\title{
A Digitized Recording Data Analysis Method for Airborne Radar
}

\author{
Surong Jiang \\ Institute of the AFEA \\ Wuhan, China \\ jiangsr@gmail.com
}

\author{
Jiangqiao Lan \\ Institute of the AFEA \\ Wuhan, China \\ LJQ@yeah.net
}

\author{
Yuhai Yang \\ Institute of the AFEA \\ Wuhan, China \\ yyhandy@yeah.net
}

\begin{abstract}
Fly detection method using for airborne pulse Doppler phased array early warning radar detection range performance evaluation is complex, expensive and timeconsuming task. In order to comprehensively evaluate the radar detection performance, it needs many data statistic independently. Inspection fly routes also need to be taken into account carefully beforehand, and reasonable to arrange. Considering the characteristics of the modern weapons and equipment, we use analysis method for their digital recording data. After corresponding screening, filtering, and sorting, and finally using the maximum likelihood estimation, we can arrive at a more precise result with some simple statistical calculations. A new way is opened up for performance evaluation of airborne pulse Doppler phased array early warning radar detection range.
\end{abstract}

Keywords-Airborne radar; detection range; digital recording data; analysis methods

\section{INTRODUCTION}

Although the low pulse repetition frequency radar no distance blur, you can distinguish between target and clutter based on distance, but there are many Doppler blind, speed performance is poor. Low pulse repetition frequency radar can not distinguish target echo from the main lobe clutter. It can only be used depending on the probe. Although the high pulse Doppler radar has good speed capability, but generally not for the distance measurement. In the use of complex ranging technology, there will still be a few kilometers from the precision error. Modern airborne early warning radar often uses the medium pulse repetition frequency. Although the pulse repetition frequency radar has both distance and speed blur, while the use of a multi-varied repetition frequency technology, we can better solve the problem of distance and speed blur. And it has both high and low repetition frequency characteristics can provide full height, full good detection performance, so it has been widely used.

Pulse Doppler radar using pulse compression technique to achieve a low narrow pulse peak power emitted, and also allows the distance to the target has a good resolution. Owing to the use of phased array antenna system of the radar and the target according to the working environment and the distribution of time, space and energy resources adaptive management, beam scheduling, fast and flexible control, to different targets and scanning area while using different detection mode, to achieve optimal use of resources and the optimal target detection, tracking results. By using phased array antenna amplitude and phase weighting techniques, the radar transmitter can achieve a low side lobe performance. At the same time, the radar receiver will use the side lobe blanking technology. Thus will reduce strong interference and increase the target detection performance.

Since the pulse Doppler radar data processing technological breakthroughs and updates, as well as a phased array antenna to match, is now used in China's AWACS airborne early warning radar. The distance evaluation for airborne early warning radar is of great significance.

\section{Fly Detection Method For Range Detection}

Radar detection range is closely related with many performance conditions. Such as carrier aircraft flight altitude, the number of false alarms, clutter characteristics, target characteristics, and so on. Airborne pulse Doppler phased array early warning radar had both pulsed Doppler system characteristics and phased array antenna system characteristics. And each system has its advantages and disadvantages. Radar designers weaknesses based on their technical principle, to carry out radar design work. Radar detection range of assessment required to carry out relevant analysis and evaluation work considering with the technical characteristics of the radar itself. Generally need to consider the following three aspects:

\section{A. Radar antenna performance}

Phased array radar relies on the transmitted signal phase changes of each unit of antenna array to control the beam scanning. Antenna beam width and gain performance increases with the scan angle decreases, while the pulse Doppler radar can increase the transmit power and the impulse in this direction to increase the number of coherent integration in order to compensate for their detection performance on decline. In order to verify the performance of the radar in this regard, the probe data of each antenna 
scan angle must be process with classification statistics for performance comparison.

\section{B. The Clutter}

For the pulse repetition frequency Doppler radar, mainly in the side lobe clutter zone detection target, target signal contend with noise and clutter. Although the same radar front, the same antenna scan angle, but the target radial velocity relative to the carrier aircraft causes the target echo position in the frequency domain is not the same. Of course, the probability of detection is different. Similarly, different fronts, the same antenna scan angle, because the environment is not the same clutter, detection performance is different. And different from the actual inspection fly zone, ground clutter strength are not the same, so we must make a comprehensive comparison of the above, on radar detection range performance assessment. The actual inspection fly zone and radar ground clutter factor performance criteria stipulated clutter factor for conversion.

\section{RCS of the target aircraft}

In different viewing angle of the target, the target's radar cross section is not the same. U.S. researchers have used B26 bomber made experiments in the laboratory . Experimental results show that a different viewing angle, the RCS values are different. The aircraft's nose and wings, and the engine will be greater impact on the target reflection cross-sectional area, as shown in Figure 1. Therefore, when evaluating the radar detection range required in the actual flight conditions depending on the angle of the radar target detection RCS values under the radar performance conditions agreed with the objectives RCS values are translated. After this, we can obtain the maximum radar detection range values in the direction.

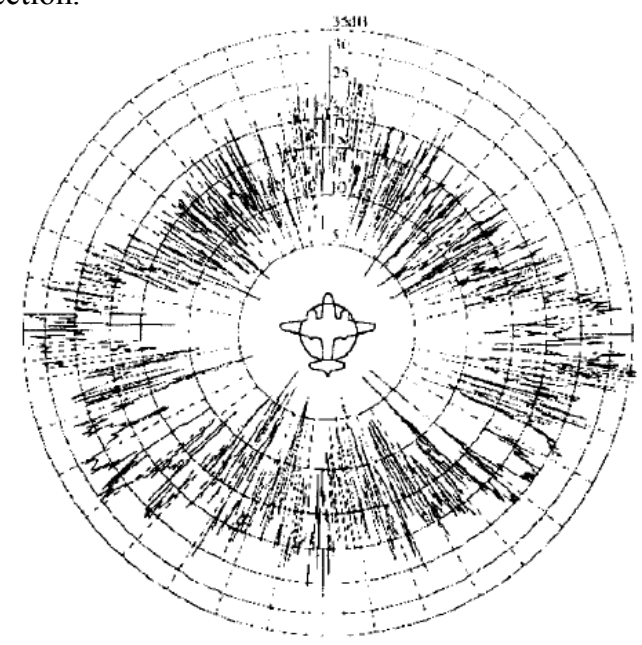

Figure 1. B26 bombers RCS pattern (the wavelength of $10 \mathrm{~cm}$ )

Fly in the inspection process, the routes design for carrier aircraft and target aircraft is a difficult problem. Vehicles seized in the development of multi-fly program, the target aircraft and carrier aircraft radar corresponding positions to cover all fronts, each frequency point, each antenna scan angle, to the back stop, and target aircraft flying height of each inspection. Because the radar system based on PD, the above conditions of the target echo clutter and target RCS of aircraft are very different, so the detect data should respectively independent statistical classification evaluation.

When select the check flight routes, in order to verify the specific radar antenna scan angle detection capability, usually containing carrier aircraft and the target aircraft on the route in their respective flight, in accordance with the method of $\mathrm{K}$, to keep pace entering into the working distance of the radar mode the maximum detection range (index value) of $110 \%$. Seized by controlling the flying speed of carrier aircraft and target aircraft, resulting in different fronts, different antenna scan angle corresponding to the target aircraft depending on the viewing angle. By carrier aircraft altitude wind, there will be a larger flight drift angle. So correction method is that the actual carrier aircraft during the flight keep the course, do not repair the wind flying, and the target aircraft keep flight path.

Airborne early warning radar belongs to the intelligence radar category, and therefore the target detection probability requirements will not be as precise as tracking radar, and we generally take detection range that the found probability of target of $50 \%$ as a maximum detection range, and for Midrange early warning radars with more than two vertical beams generally take $20 \mathrm{~km}$ distance intervals sampled.

To increase sample values confidence, adjacent sampling intervals can overlap distance of $1 / 2$ for step, then with the probability, according to the requirements of developing confidence intervals and confidence level, determine the distance interval observation points required to facilitate inspection fly sorties calculations. If according to the probability of detection of $50 \%$, confidence level of $90 \%$, the half-width of the confidence interval 0.092, then the observation will need 80 points.

Since the entry angle of the target aircraft is different, and the carrier aircraft relative radial velocity is not the same, so the radar in their detection distance interval is not the same number of points, the entry angle of each target aircraft flying sorties into the check are also different

Through the actual fly inspection, under the same detect conditions, corresponding to the distance of each inspection fly sorties probe segment number and frequency were found to accumulate, to obtain the total number of probes and found that the number of segments to calculate the probability of detection of each value from the last, the entire detection range of the distance between the probability of curve fitting software to fit the curve corresponds to the probability to $50 \%$ of the distance from the detection probability for the particular conditions of maximum detection distance of the radar detection. Then according to the conditions stipulated in radar performance coefficient of ground clutter and target detection range aircraft RCS values are translated on to get the final detection distance values.

\section{Digital Recording Data Analysis Methods}

It is not a simply calculation for data accumulate when get the detection capability by digitizing airborne radar recorded data. The first is to go through scientific screening. Screened the real fly data that meet statistical requirements. 
The first round of screening is classification filtering. For early warning aircraft, the low and high altitude detection results are different. Under normal circumstances, it can be divided into some height layers. So the first time statistical classification is from a height level of classification. The second segment is the RCS of the target aircraft radar. There is a transformation. Attributes section aircraft types have been identified for these known models, index by its RCS. After this transformation, target RCS characteristics can be classified.

The second round of screening is the target aircraft flight attitude filtering. Records from airborne radar data, parse the target aircraft's speed and heading data to calculate its AWACS angle between the heading and the radial velocity. AWACS radial screened out for the next target flight statistics.

The next important step is to overlay. It will go through two filtered data as a whole to consider, calculated that the probability of the maximum distance greater than a specified value. Three fronts of the airborne radar's performance is approximate, while airborne radar antenna array surface normal centered symmetrically on both sides of the position is similar performance. If you find an individual orientation fewer dots, it generally corresponding airspace really no goal. And therefore can be normal along the center of each antenna array surface extrapolation between the front and three simple extrapolation to obtain the extrapolated value. The valuation of the real fly in a large number of data revision process will tend to keep a true value.

Finally, each calculation as an experiment, based on the maximum likelihood principle, using the maximum likelihood estimation method, calculated every time a "likelihood" curve, in which the detection probability is greater than the specified value selected distance set, constitute the actual probe distance curve. Repeat the process until the last three actual detection distance is less than the difference between the curves given value $\Delta \mathrm{Z}$, you can terminate the data overlay process to obtain maximum detection distance curve. This approach has four advantages:

- It is simple. It does not depend on the test with flying, and the measurement according to the real need only flying flight data and associated auxiliary data, these data are basic data retained when flying, without the need for complex measurements of various parameters.

- It can for a certain type, or even for one aircraft make a early warning aircraft performance measurement, measurement results compared to the results of the background technology, as are used in the actual data, and thus more accurate, and high level of confidence degrees can be achieved after a period of the total time.

- Compare with test aircraft flight test methods, because you do not make special arrangements with the flight test aircraft, it is usually rely on the data accumulated naturally flight, and without the need to pay extra cost for arrange test flying, so the aircraft performance measurement of airborne radar has lower cost.

- Only need to download the data to a computer on the ground, do not need to make any changes for the AWACS and specialized parameter measurement, perform the appropriate software, it can be realized.

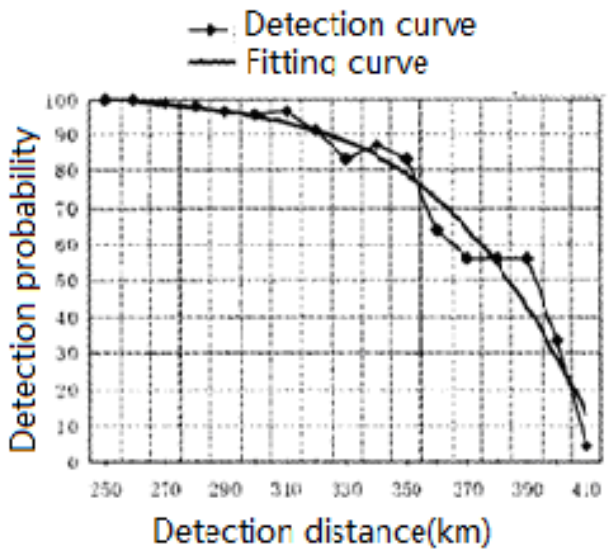

Figure 2. Airborne early warning radar recording data analysis method detection probability fitting diagram

\section{SUMMARY}

Fly detection method using for airborne pulse Doppler phased array early warning radar detection range performance evaluation is a complex and time-consuming task that requires many independent statistical data in order to comprehensively evaluate the radar detection performance. Using recording data analysis method, relatively speaking only need to analysis the AWACS mission system records data and do some statistical computing; we can arrive at a more precise result. Thus opens up a new performance evaluation methods for airborne pulse Doppler phased array early warning radar detection range.

\section{REFERENCE}

[1] Dai Chengsong. Airborne pulse Doppler radar detection performance fly [J]. Modern radar, 1998,20 (1), pp.68-73.

[2] Zhang Guangyi. Phased array radar system [M]. Beijing: National Defense Industry Press, 1994

[3] Xiong Jiajun, etc. Airborne early warning radar intelligence analysis technology and application [M]. Beijing: Military Yiwen Publishing House, 2013.

[4] Yao Huafei. Airborne pulse Doppler phased array early warning radar detection range performance evaluation $[\mathrm{J}]$. Electro-optical system, 2008,3 (1), pp.14-16..

[5] Merrill I. Skolnik. Radar Handbook(Second Edition) [M], New York:McGraw-Hill,2003. 